\title{
Reconstruction of Fiber Trajectories via Population-Based Estimation of Local Orientations
}

\author{
Pew-Thian Yap ${ }^{1}$, John H. Gilmore ${ }^{2}$, Weili Lin ${ }^{1}$, and Dinggang Shen ${ }^{1}$ \\ 1 BRIC, Department of Radiology and \\ 2 Department of Pyschiatry \\ University of North Carolina at Chapel Hill, NC \\ \{ptyap, jgilmore, weili_lin, dgshen\}@med.unc.edu
}

\begin{abstract}
White matter fiber tractography plays a key role in the in vivo understanding of brain circuitry. For tract-based comparison of a population of images, a common approach is to first generate an atlas by averaging, after spatial normalization, all images in the population, and then perform tractography using the constructed atlas. The reconstructed fiber trajectories form a common geometry onto which diffusion properties of each individual subject can be projected based on the corresponding locations in the subject native space. However, in the case of High Angular Resolution Diffusion Imaging (HARDI), where modeling fiber crossings is an important goal, the above-mentioned averaging method for generating an atlas results in significant error in the estimation of local fiber orientations and causes a major loss of fiber crossings. These limitatitons have significant impact on the accuracy of the reconstructed fiber trajectories and jeopardize subsequent tract-based analysis. As a remedy, we present in this paper a more effective means of performing tractography at a population level. Our method entails determining a bipolar Watson distribution at each voxel location based on information given by all images in the population, giving us not only the local principal orientations of the fiber pathways, but also confidence levels of how reliable these orientations are across subjects. The distribution field is then fed as an input to a probabilistic tractography framework for reconstructing a set of fiber trajectories that are consistent across all images in the population. We observe that the proposed method, called POPTRACT, results in significantly better preservation of fiber crossings, and hence yields better trajectory reconstruction in the atlas space.
\end{abstract}

\section{Introduction}

Diffusion Tensor Imaging (DTI) is a powerful imaging modality that allows probing into the intricate micro-architecture of white matter. It plays an indispensable role in characterizing neural pathways in vivo by means of fiber tractography, which entails reconstructing the trajectories of fiber paths by tracing the direction of maximal water diffusion. Such estimated fiber paths can subsequently be used, for instance, to investigate brain connectivity alterations in 
mental and neurological disorders. Tract pathology can be evident, as in the case of brain tumors where tracts are grossly displaced, or subtle, as in the case of neuropsychiatric disorders, such as schizophrenia where disruptions are manifested as change of diffusion properties within the tracts.

The core assumption of DTI - Gaussianity of water diffusion - however, does not always hold true. Modeling water diffusion using the single tensor formulation ignores this complexity and results in loss of information. One of the methods proposed to remedy the shortcomings of DTI is High Angular Resolution Diffusion Imaging (HARDI) [1, where diffusion signals are acquired along a significantly larger number of directions than is normally employed in DTI. This allows modeling of the complex non-Gaussian diffusion process and construction of spherical functions with multiple local maxima which are potentially aligned with the underlying fiber bundle orientations. Accordingly, one naturally wants to extend existing DT-based tractography algorithms to work with HARDI data to better deal with fiber crossings and to reconstruct fiber trajectories that resemble more closely the anatomical fibers connecting different functional regions of the brain.

In this paper, we detail a tractography algorithm, called POPTRACT, that allows effective reconstruction of fiber trajectories in the atlas space by more faithfully preserving fiber crossings. Tractography in the atlas space, as was done in previous works [2, allow the reconstructed trajectories to form a common geometry onto which diffusion properties from the individual images can be projected for tract-based comparison. To this end, a common step involves averaging the tensors [2], the Fiber Orientation Distributions (FODs) [3], or the diffusion signals [4 after spatial normalization, resulting in an atlas on which tractography can be performed after determining the local fiber orientations. We will show, however, that this approach causes significant deviation of the estimated local orientations from the 'true' orientations. The major reason for this is the smearing of the orientation profile, caused by averaging misaligned ODFs.

\section{Approach}

The main idea of our approach is to model the orientation distribution at each voxel location by simultaneously considering all images in the population. Similar to the average atlas approach, we want to use this orientation information to reconstruct a set of fiber trajectories that will form a common datum, based on which inter-subject tract-based morphometry can be performed. Unlike the average atlas approach, however, orientations are not estimated after, but prior to, averaging in the common space to avoid estimation inaccuracy caused by ODF smearing. The estimated orientation fields are transformed to a common atlas space where the maximum likelihood parameter estimates of the Watson distribution at each voxel location are determined. A probabilistic tractography algorithm is then be used to reconstruct the fiber trajectories. 


\subsection{Modeling Local Fiber Orientations}

Assuming for a moment that the orientation fields computed from $N$ diffusionweighted images are spatially normalized to a common space and that each voxel location of an orientation field contains only one fiber orientation, our goal now is to accurately relate these orientations to an underlying model. At each voxel location of orientation field $i \in\{1, \ldots, N\}$, the local fiber orientation is denoted by a unit vector $\mathbf{v}_{i}$. We model the distribution of orientations across subjects using the bipolar Watson distribution, probability density function (PDF) of which is given by [5] $f(\mathbf{v} \mid \boldsymbol{\mu}, \kappa)=C(\kappa) \mathrm{e}^{\kappa\left(\boldsymbol{\mu}^{\mathrm{T}} \mathbf{v}\right)^{2}}$. The parameter $\boldsymbol{\mu}$ is a unit vector called the mean orientation and $\kappa$ is a positive constant called the concentration parameter. The density has maxima at $\pm \boldsymbol{\mu}$ and becomes more concentrated around $\pm \boldsymbol{\mu}$ as $\kappa$ increases. The density is also rotationally invariant around $\pm \boldsymbol{\mu}$. $C(\kappa)$ is a normalizing constant to ensure that the density function integrates to unity over the unit sphere.

Parameter Estimation. (Single Fiber Orientation) Assuming that the orientation vectors obtained from the images, $\mathbf{v}_{1}, \ldots, \mathbf{v}_{N}$, are random samples from the Watson distribution, the maximum likelihood estimate (MLE) of $\boldsymbol{\mu}$ is the eigenvector corresponding to the largest eigenvalue $\lambda_{1}\left(\lambda_{1} \geq \lambda_{2} \geq \lambda_{3}\right)$ of the symmetric positive definite matrix [5] $\mathbf{A}=\frac{1}{N} \sum_{i=1}^{N} \mathbf{v}_{i} \mathbf{v}_{i}^{\mathrm{T}}$. Matrix $\mathbf{A}$ is called the dyadic tensor. The MLE of $\kappa$ is $\left(1-\lambda_{1}\right)^{-1}$, asymptotically when $\kappa \rightarrow \infty[5]$. When $\kappa=0$, the distribution is uniform. As $\kappa$ increases, the PDF becomes more concentrated about $\pm \boldsymbol{\mu}$. Therefore, orientations that depart from $\pm \boldsymbol{\mu}$ are penalized more heavily when there is a strong alignment of the local fiber orientations from all subjects. (Extension to Multiple Fiber Orientations) Denoting the maximum number of possible orientations as $\Omega$, we now denote the set of direction vectors at each voxel as $\left\{\mathbf{v}_{i}^{[D]}\right\}$, where $D=1, \ldots, \Omega$. In the case where there is less than $D$ orientations, the surplus orientation vectors are simply set to nil. For DTI, $\Omega$ is limited to 1 . For HARDI, however, the representation models used often allows more than one fiber orientations, hence $\Omega \geq 1$. Assuming the presence of multiple compartments in each voxel, each representing the local structure of a fiber bundle, and that there is no exchange between the different compartments, we estimate the parameters of the PDF for each compartment independently. That is, for compartment $D$, we estimate $\boldsymbol{\mu}^{[D]}$ and $\kappa^{[D]}$ using $\mathbf{v}_{1}^{[D]}, \ldots, \mathbf{v}_{N}^{[D]}$.

\subsection{Reconstructing Fiber Trajectories}

A white matter fiber can be modeled as a finite-length path parameterized by a train of unit length vectors. We use the following notation for such a path: $\mathbf{v}_{(1: T)}=\left\{\mathbf{v}_{(1)}, \ldots, \mathbf{v}_{(T)}\right\}$. We further assume that a fiber path can be traced by tracking the trajectory of a particle traveling in an orientation field. Each particle is endowed with an initial speed in an appropriate direction. It then moves with constant speed to position $\mathbf{p}_{(t)}$ according to $\mathbf{p}_{(t+1)}=\mathbf{p}_{(t)}+s \mathbf{v}_{(t)}$, where $s$ is the step length. At each point in space, vector $\mathbf{v}_{(t)}$ is drawn from distribution 
$f\left(\mathbf{v}_{(t)} \mid \mathbf{v}_{(t-1)}, \boldsymbol{\theta}_{(t)}, D_{(t)}\right)$, where the set of parameters for the Watson distributions are collectively denoted as $\boldsymbol{\theta}_{(t)}=\left\{\boldsymbol{\mu}_{(t)}^{[1]}, \ldots, \boldsymbol{\mu}_{(t)}^{[\Omega]}, \kappa_{(t)}^{[1]}, \ldots, \kappa_{(t)}^{[\Omega]}\right\}$. The above distribution is in fact the Watson distribution discussed in Section 2.1. It is, however, now dependent on the prior orientation vector $\mathbf{v}_{(t-1)}$ in determining which orientation compartment $D_{(t)}=1, \ldots, \Omega$ to follow in the case where a voxel contains multiple orientations. Assuming that the Watson distribution is not directly dependent on $\mathbf{v}_{(t-1)}$ but only on $D_{(t)}$, we can simplify $f\left(\mathbf{v}_{(t)} \mid \mathbf{v}_{(t-1)}, \boldsymbol{\theta}_{(t)}, D_{(t)}\right)$ to become $f\left(\mathbf{v}_{(t)} \mid \boldsymbol{\theta}_{(t)}, D_{(t)}\right)$. Specifically,

$$
f\left(\mathbf{v}_{(t)} \mid \mathbf{v}_{(t-1)}, \boldsymbol{\theta}_{(t)}, D_{(t)}\right)=f\left(\mathbf{v}_{(t)} \mid \boldsymbol{\theta}_{(t)}, D_{(t)}\right)=C\left(\kappa_{(t)}^{\left[D_{(t)}\right]}\right) \mathrm{e}^{\kappa_{(t)}^{\left[D_{(t)}\right]}\left(\left(\boldsymbol{\mu}_{(t)}^{[D(t)]}\right)^{\mathrm{T}} \mathbf{v}_{(t)}\right)^{2}}
$$

and

$$
f\left(D_{(t)} \mid \mathbf{v}_{(t-1)}, \boldsymbol{\theta}_{(t)}\right) \propto \begin{cases}\bar{\rho}_{(t)}^{\left[D_{(t)}\right]}\left[\mathbf{v}_{(t-1)}^{\mathrm{T}} \boldsymbol{\mu}_{(t)}^{\left[D_{(t)}\right]}\right]^{2}, & \left|\mathbf{v}_{(t-1)}^{\mathrm{T}} \boldsymbol{\mu}_{(t)}^{\left[D_{(t)}\right]}\right| \geq \cos (\phi) \\ 0, & \text { otherwise }\end{cases}
$$

where $\phi$ is the maximum allowed turning angle and $\bar{\rho}_{(t)}^{[D]}$ is defined as $\bar{\rho}_{(t)}^{[D]}=$ $\frac{1}{N} \sum_{i=1}^{N} \rho_{i,(t)}^{[D]}$, with $\rho_{i,(t)}^{[D]}$ being the value of the ODF at the voxel location determined by $\mathbf{p}_{(t)}$, sampled at orientation $\mathbf{v}^{[D]}$. The tracing is stopped if the trajectory reaches a voxel with orientation coherence $\beta^{[D]}=1-\sqrt{\frac{\lambda_{2}^{[D]}+\lambda_{3}^{[D]}}{2 \lambda_{1}^{[D]}}}$, $\beta^{[D]} \in[0,1]$ falling below a predefined threshold $\beta_{0}$, or simply when the brain boundary is encountered. The $\lambda$ 's are the eigenvalues of the dyadic tensor at each voxel location. Perfect alignment of the orientations in compartment $D$ results in $\beta^{[D]}=1$ and an uniform distribution of orientations results in $\beta^{[D]}=0$.

\section{Results}

\subsection{Synthesized Dataset}

To evaluate the effectiveness of the proposed method in preserving fiber crossings and in correctly estimating the orientations, we synthesized an $8 \times 8$ image (Camino [6]; two-tensor model) with each voxel containing a crossing with two orientations - one vertical and one horizontal. To simulate inter-subject variability, we perturbed the synthesized diffusion-weighted signals by applying random rotation matrices (angles: $15^{\circ}, 30^{\circ}, 45^{\circ}, 60^{\circ}$ ) and adding isotropic complex Gaussian noise $(\mathrm{SNR}=8,16)$ to each voxel. The ODF at each voxel is then computed using Camino [6]. We applied these perturbations 10 times to the no-noise image and then used the resultant images to attempt to recover the 'true' populationbased orientations. We show, for qualitative evaluation, the results for the case of $45^{\circ}$ and $\mathrm{SNR}=16$ in Fig. 1. It can be observed that the average atlas method (Fig. 1 (b)), generated by averaging the respective ODFs, resulted in loss of fiber crossings and significant deviation in the estimated orientations. The proposed method (Fig. 1(c)) gave a more consistent result. 


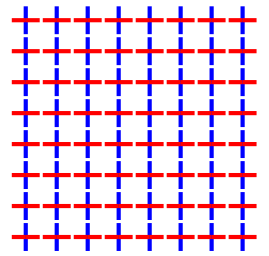

(a) Ground Truth

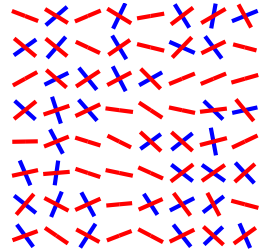

(b) Average Atlas

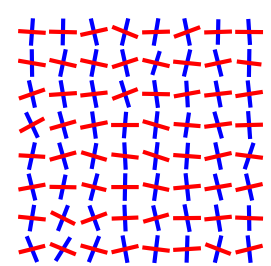

(c) PopTract

Fig. 1. Estimation of orientations using different schemes

For quantitative evaluation, we measured the orientational discrepency (OD) of the estimated orientations with respect to the ground truth orientations. Assuming that $\mathbf{U}(\mathbf{x})$ is the set of ground truth directions at voxel location $\mathbf{x}$ and $\mathbf{V}(\mathbf{x})$ is the corresponding set of estimated directions, OD is defined as

$$
\mathrm{OD}(\mathbf{x})=\frac{1}{2}\left[\max _{\mathbf{u} \in \mathbf{U}(\mathbf{x})} \min _{\mathbf{v} \in \mathbf{V}(\mathbf{x})} d_{\theta}(\mathbf{u}, \mathbf{v})+\max _{\mathbf{v} \in \mathbf{V}(\mathbf{x})} \min _{\mathbf{u} \in \mathbf{U}(\mathbf{x})} d_{\theta}(\mathbf{v}, \mathbf{u})\right]
$$

where $d_{\theta}(\mathbf{u}, \mathbf{v})$ gives the angle difference between $\mathbf{u}$ and $\mathbf{v}$, i.e., $d_{\theta}(\mathbf{u}, \mathbf{v})=$ $\cos ^{-1}(|\mathbf{u} \cdot \mathbf{v}|)$. The absolute value is taken since diffusion is assumed to be antipodal symmetric. In cases of multiple local maxima, the term $\min _{\mathbf{v} \in \mathbf{V}} d_{\theta}(\mathbf{u}, \mathbf{v})$ returns the angle difference between $\mathbf{u}$ with an orientation $\mathbf{v}$ in $\mathbf{V}$ that is most closely aligned with itself. Evaluating the OD between the estimated orientations with the ground truth under different rotation angles and SNRs, the results, shown in Fig. 2, indicate that the proposed method is capable of estimating orientations which are closer to the ground truth, with improvement especially prominent when the angles of rotation is large.

SNR $=8$

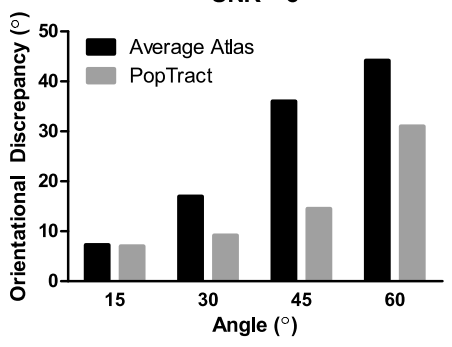

SNR $=16$

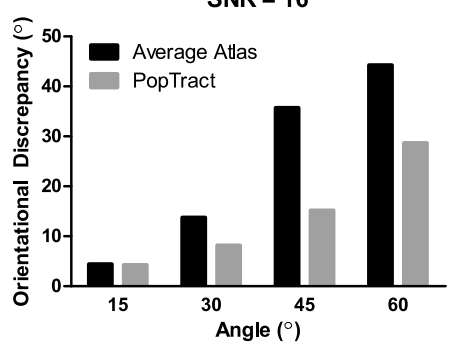

Fig. 2. Orientational dicrepancy between the estimated orientations and the ground truth orientations under different rotation angles and signal-to-noise ratios.

\subsection{In Vivo Dataset}

Materials. Diffusion-weighted images were acquired for 14 adult subjects using a Siemens 3T TIM Trio MR Scanner with an EPI sequence. Diffusion 


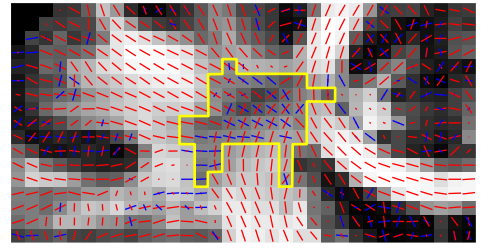

(a) Template

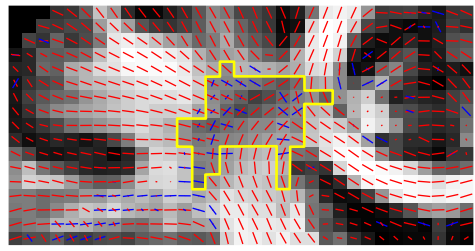

(b) Average Atlas

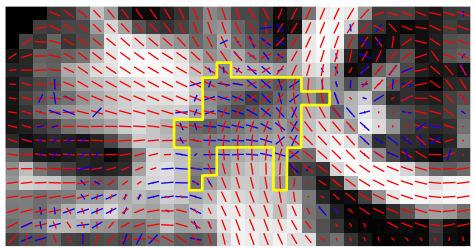

(c) PopTract

Fig. 3. Fiber crossings. A significant loss of crossings can be observed for the average atlas method.

gradients were applied in 120 non-collinear directions with diffusion weighting $b=2000 \mathrm{~s} / \mathrm{mm}^{2}$, flip angle $=90^{\circ}$, repetition time $(\mathrm{TR})=12,400 \mathrm{~ms}$ and echo time $(\mathrm{TE})=116 \mathrm{~ms}$. The imaging matrix was $128 \times 128$ with a rectangular FOV of $256 \times 256 \mathrm{~mm}^{2} .80$ contiguous slices with a slice thickness of $2 \mathrm{~mm}$ covered the whole brain. Data post-processing includes brain skull removal, motion correction and eddy current correction using algorithms developed and distributed as part of the FMRIB Software Library (FSL) package.

Spatial Normalization and Orientation Estimation. The local maxima of an ODF reflect local fiber orientations. In our case, the orientations were computed with Camino 6] by locating the peaks of the ODFs using Powell's algorithm on a set of sample points generated by randomly rotating a unit icosahedron 1000 times. We limited the maximum number of orientations for each voxel to two. This choice is guided by several previous studies [7,8], where the estimation of two orientations is generally deemed as stable. One image was selected, out of the 14 images, as the template onto which 13 other images were registered using a deformable spherical-harmonics-based HARDI registration algorithm [9] to align the ODFs. The estimated transformations were used to map the respective orientation fields to a common space. When transforming the orientations, they were reoriented using $\mathbf{v}^{\prime}=\mathbf{F} \mathbf{v} /\|\mathbf{F} \mathbf{v}\|$, where $\mathbf{F}$ is a local affine transform matrix computed from the image transformation map. For generating the average atlas, transformation of the ODF was performed based on a method similar to that proposed by Rafflet et al. [3] we approximated the ODF using a number of Point Spread Functions (PSFs), reoriented these PSFs individually, and then recomposed the reoriented PSFs to obtain the transformed ODF. The 


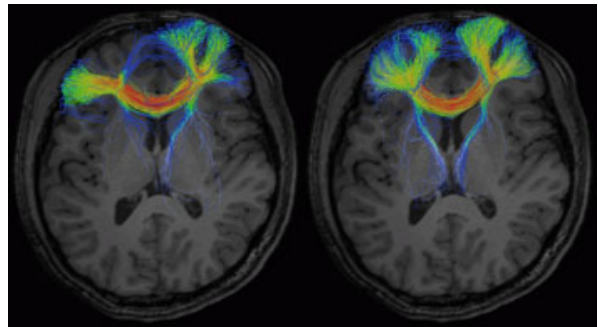

(a) Forceps Minor

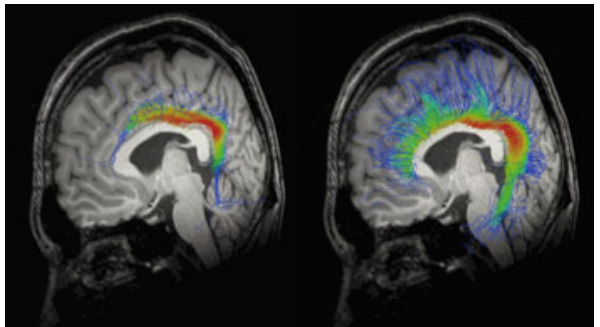

(b) Cingulum Bundle

Fig. 4. Fiber trajectory reconstruction of the (a) forceps minor and (b) cingulum bundle. The results given by the average atlas method and POPTRACT are shown on the left and right, respectively. The coloring indicates the probability of finding a fiber at a specific spatial location. Dark red indicates a high probability of a particular location being traversed by fibers, and dark blue indicates otherwise.

same exact set of deformation fields, as applied to the orientation fields, were used to align the ODF fields. The average ODF at each voxel was then used to estimate the local fiber orientations.

Crossings. Similar to the results shown for the synthesized data (Fig. 1), significant loss of fiber crossings can be observed in the case of the in vivo data when the average atlas method is used for estimating the orientations (Fig. 3).

Tractography. For evaluation, we considered the following commonly studied fiber bundles: 1) The forceps minor, also known as the anterior forceps, is a fiber bundle which connects the lateral and medial surfaces of the frontal lobes and crosses the midline via the genu of the corpus callosum; 2) The cingulum is a medial associative bundle that runs within the cingulate gyrus all around the corpus callosum. To test whether these fiber bundles were preserved by the proposed method, we placed single voxel seeds at the points where the midline crosses the genu of the corpus callosum, and also a seed along the cingulate gyrus, and performed tractography based on these seeds. The tractography parameters used were $\phi=70^{\circ}, \beta_{0}=0.1$ and $s=1.3000$ fibers were initiated from each seed. An identical set of parameters was used for the average atlas method. The results, shown in Fig. 4, indicate that the average atlas method results in premature termination of the tracking of the fiber bundles. Specifically, in Fig. 4(a), we find that PopTRACT, as opposed to the average atlas method, not only reconstructed the full forceps minor, with both frontal extensions of the fiber bundles intact, but also part of the anterior thalamic radiations. Similar conclusion can be made from Fig. 4(b), where PopTRACT shows a complete reconstruction of the cingulum bundle and the U-shaped fibers connecting the medial frontal, parietal, occipital and temporal lobes with different portions of the cingulate cortex. POPTRACT, therefore, in contrast to the conventional average atlas method, gives a more complete reconstruction of the fiber trajectories, which are more consistent with our anatomical knowledge of the bundles. 


\section{Conclusion}

We have presented a tractography algorithm, called POPTRACT, that is more effective in preserving fiber crossings and is more accurate in estimating local fiber orientations. POPTRACT results in more reasonable reconstruction of the fiber trajectories that are in closer agreement with known fiber bundles.

Acknowledgment. This work was supported in part by NIH grants: EB006733, EB008374, EB009634, MH088520, HD05300, MH064065, and NS055754.

\section{References}

1. Tuch, D.S., Weisskoff, R.M., Belliveau, J.W., Wedeen, V.J.: High angular resolution diffusion imaging of the human brain. In: ISMRM 1999 (1999)

2. Goodlett, C.B., Fletcher, P.T., Gilmore, J.H., Gerig, G.: Group analysis of DTI fiber tract statistics with application to neurodevelopment. NeuroImage 45(1), S133-S142 (2009)

3. Raffelt, D., Tournier, J., Fripp, J., Crozier, S., Connelly, A., Salvado, O.: Symmetric diffeomorphic registration of fibre orientation distributions. NeuroImage (2011)

4. Bouix, S., Rathi, Y., Sabancu, M.: Building an average population atlas. In: MICCAI 2010 Workshop on Computational Diffusion MRI (2010)

5. Schwartzman, A., Dougherty, R.F., Taylor, J.E.: False discovery rate analysis of brain diffusion direction maps. Annals of Applied Statistics 2(1), 153-175 (2008)

6. Cook, P.A., Bai, Y., Nedjati-Gilani, S., Seunarine, K.K., Hall, M.G., Parker, G.J., Alexander, D.C.: Camino: Open-source diffusion-MRI reconstruction and processing. In: 14th Scientific Meeting of the International Society for Magnetic Resonance in Medicine, p. 2759 (2006)

7. Tuch, D., Reese, T., Wiegell, M., Makris, N., Belliveau, J., Wedeen, V.: High angular resolution diffusion imaging reveals intravoxel white matter fiber heterogeneity. Magnetic Resonance in Medicine 48, 577-582 (2002)

8. Peled, S., Friman, O., Jolesz, F., Westin, C.F.: Geometrically constrained twotensor model for crossing tracts in DWI. Magnetic Resonance Imaging 24(9), 1263-1270 (2006)

9. Yap, P.T., Chen, Y., An, H., Yang, Y., Gilmore, J.H., Lin, W., Shen, D.: SPHERE: SPherical Harmonic Elastic REgistration of HARDI data. NeuroImage 55(2), 545556 (2011) 